\title{
ELISA for toxoplasma antibody detection: a comparison with other serodiagnostic tests
}

\author{
A BALSARI,* G POLI,* V MOLINA, $\dagger$ M DOVIS, $\ddagger$ E PETRUZZELLI, $\ddagger$ \\ A BONIOLO, $\S$ AND E ROLLERI
}

From the *Istituto di Microbiologia e Immunologia Veterinaria dell'Università, Via Celoria 10, Milan; $\dagger$ Centro Analisi Mediche, Piazza Trente e Trieste, Monza; $\ddagger$ SORIN Biomedica, Saluggia (VC); $\S$ Clinical Immunochemistry Unit SORIN Biomedica-Laboratorio Fisiologia Clinica CNR, Saluggia and Pisa, Italy

SUMMARY An ELISA method was developed for the measurement of toxoplasma IgG antibodies in human serum using antigen-coated polystyrene beads as a solid phase and anti human IgG-horse radish peroxidase conjugate as an enzymatic tracer. In order to assess ELISA sensitivity and specificity, a between methods comparison was made using 'conventional' serological tests as reference (dye-test, crossover-linked immunoassay, passive haemagglutination, indirect immunofluorescence). From an analysis of the group classifications obtained some considerations emerged: the ELISA specificity looks comparable with that of the 'reference' tests, as no sample classified as negative by all these tests was ELISA-positive, and vice versa; ELISA appears to correlate better with haemagglutination and immunofluorescence, on the basis of the respective class frequencies; in particular, the number of positives, which is much lower for the dye-test and crossover-linked immunoassay, suggests that a higher sensitivity is reached in the former cases.

None of the several tests mostly used for the serodiagnosis of toxoplasmosis appears fully adequate for mass-screening purposes as far as analytical reliability, experimental ease, and promptness of response are concerned. As a matter of fact some of them need special equipment not widely available, and others cannot be used as single tests owing to the incompleteness of the information obtainable and the poor correlation with the clinical situation.

Recently, the enzyme-linked immunosorbent assay (ELISA) has been proposed as a promising serological test for infective and infestive diseases. ${ }^{1-5}$

Also in our laboratories attempts were made to evaluate the actual potential of ELISA in the diagnosis of toxoplasma infections. In particular, this study was aimed at defining the specificity and sensitivity of an ELISA method we have recently developed through a comparison with other serological tests assumed as references, such as the dyetest, crossover-linked immunoassay, indirect haemagglutination, and indirect immunofluorescence.

Received for publication 6 December 1979

\section{Material and methods}

SAMPLES

The samples were taken at random among human sera sent for toxoplasma serodiagnosis to diagnostic centres in Milan.

ELISA REAGENTS AND PROCEDURE

\section{Antigen}

Soluble extracts from throfozoites of Toxoplasma gondii, RH strain, harvested from the peritoneal exudate of mice 72 hours after injection, were prepared by sonication disruption of cells, followed by centrifugation. A solution of $10 \mu \mathrm{g}$ protein $/ \mathrm{ml}$ in Tris- $\mathrm{HCl}$ buffer, $\mathrm{pH} 7 \cdot 2$, was used for coating.

\section{Solid phase}

Polystyrene beads, ca $6 \mathrm{~mm}$ diameter (Precision Plastic Balls, USA), were sensitised through overnight immersion in the coating solution at room temperature, followed by washing with deionised water and vacuum-drying. 


\section{Enzyme-labelled anti human globulins}

Horse radish peroxidase (HRP, Boehringer, FRG) and rabbit anti human IgG, purified by affinity chromatography, were coupled using the periodate method $^{6}$ slightly modified to obtain an approximate HRP/IgG molar ratio of 2 . The conjugate was purified through gel-filtration on Sephadex G-200 (Pharmacia, Sweden) and used in the assay at a dilution of approximately $0.4 \mu \mathrm{g} / \mathrm{ml}$ in $0.05 \mathrm{M}$ phosphate buffer, $\mathrm{pH} 7 \cdot 4$, containing $4 \%$ bovine serum albumin (BSA) and $0.01 \%$ merthiolate.

\section{Substrate for the enzymatic reaction}

$150 \mathrm{mg}$ of $O$-phenylene-diamine dihydrochloride (home-prepared from the free base) were added before use to $50 \mathrm{ml}$ of $0.25 \mathrm{M}$ phosphate-citrate buffer containing $0.02 \% \mathrm{H}_{2} \mathrm{O}_{2}$, to obtain a final $\mathrm{pH}$ of $4 \cdot 8$.

\section{Diluent system for serum samples}

$0.05 \mathrm{~m}$ phosphate buffered saline, $\mathrm{pH} \mathrm{7.4,4 \%}$ BSA, $0.01 \mathrm{~m}$ EDTA, $0.1 \% \mathrm{NaN}_{3}$, was used.

\section{ELISA procedure}

The following scheme was adopted:

-1st incubation: test solution $15 \mathrm{~min}, 40^{\circ} \mathrm{C}$

$(0 \cdot 2 \mathrm{ml} \mathrm{1:10} \mathrm{diluted} \mathrm{sample}$

for each bead)

-Washing with deionised $\quad 4 \times 2.5 \mathrm{ml}$

water

-2nd incubation with enzymatic $15 \mathrm{~min}, 40^{\circ} \mathrm{C}$ tracer $(0 \cdot 2 \mathrm{ml})$

-Washing with deionised $\quad 4 \times 2.5 \mathrm{ml}$ water

-3rd incubation with substrate $10 \mathrm{~min}$, $(0.5 \mathrm{ml})$

-Stop with $2 \mathrm{~N} \mathrm{H}_{2} \mathrm{SO}_{4}(1.0 \mathrm{ml})$

-Absorbance readings at $492 \mathrm{~nm}$

The ELISA data were expressed as ELISA mU, ie, absorbance $\times 10^{3}$.

\section{'REFERENCE' SEROLOGICAL TESTS}

The procedure reported by Sabin and Feldman ${ }^{7}$ was followed for the dye-test (DT). The passive haemagglutination test (HA) was performed using the Toxo-HA kit supplied by BioMerieux (France). For the indirect immunofluorescence (IF) test the IF test of Roche Diagnostics (USA) was used. The crossover-linked immunoassay (cLIA) was carried out according to the procedure described by Zotti et al. ${ }^{8}$ using the same antigen preparation as in the case of ELISA.

\section{Results and discussion}

Eighty-six serum samples were tested with the reference methods and accordingly classified as three distinct groups: negative, 'borderline' (ie, weakly positive), and positive. The classification criteria for each method and the resulting distribution of samples are reported in Tables 1 and 2, respectively. The latter table shows that 16 samples are classified as negative and 12 as positive by all the reference methods ('reliably' negative and 'reliably' positive).

Table 1 Classification criteria of the 'reference' tests

\begin{tabular}{lllll}
\hline $\begin{array}{l}\text { Sample } \\
\text { classification }\end{array}$ & \multicolumn{4}{l}{ Test response (reciprocal titres) } \\
\cline { 2 - 5 } & $D T$ & $c L I A^{*}$ & $H A$ & $I F$ \\
\hline Negative & $<50$ & - Undiluted & $<40$ & $<50$ \\
Borderline & $50-100$ & + Undiluted & $40-160$ & $50-100$ \\
Positive & $\geqslant 250$ & $\oplus$ 1/4 diluted & $\geqslant 320$ & $\geqslant 200$ \\
\hline
\end{tabular}

*As in this case the sera are tested undiluted, 1/4 diluted, and 1/16 diluted, the results are expressed as the maximal !dilution still corresponding to a visible precipitation.

Table 2 Classification of the samples $(n=86)$ according to the 'reference' tests

\begin{tabular}{llll}
\hline Test & Frequency & \\
\cline { 2 - 4 } & Negative & Borderline & Positive \\
\hline DT & 39 & 32 & 15 \\
CLIA & 47 & 24 & 15 \\
DT + cLIA & 35 & 16 & 12 \\
HA & 24 & 29 & 33 \\
IF & 24 & 35 & 27 \\
HA + IF & 17 & 20 & 18 \\
ALL & 16 & 0 & 12 \\
\hline
\end{tabular}

The 16 reliably negative samples gave in the ELISA system a mean value and an SD of $126 \pm 17.5 \mathrm{mU}$. Therefore, assuming a $1 \%$ probability of misclassification (ie, $\bar{x}+4$ SD), the upper limit for the negative population is $196 \mathrm{mU}$. For the 12 reliably positive samples mean and SD were $469 \pm 124 \mathrm{mU}$. This corresponds to a distribution ranging, at a $95 \%$ probability ( $\overline{\mathrm{x}}-2 \mathrm{SD}$ to $\overline{\mathrm{x}}+2 \mathrm{SD})$, from 221 to $717 \mathrm{mU}$ without any overlap with the negative class.

From an analysis of the ELISA data, imposing the $196 \mathrm{mU}$ limit for the negative population, 25 out of 86 samples were negative. A comparison of these results with those given by the other methods (Table 3 ) demonstrated that ( $a$ ) no ELISA negative sample was classified as positive by any other method, and (b) nine negatives were seen as borderline by one or two reference methods (ie, $5 \mathrm{IF}, 2 \mathrm{HA}$, and 2 cLIA borderlines).

Taking into account all the reference tests combined together, no 'reliably' borderline population can be identified (Table 2). Therefore, to complete 
Table 3 Analysis of the ELISA negatives $(n=25)$ according to the 'reference' tests

\begin{tabular}{llll}
\hline Test & Frequency & & \\
\cline { 2 - 4 } & Negative & Borderline & Positive \\
\hline DT & 25 & 0 & 0 \\
cLIA & 23 & 2 & 0 \\
HA & 23 & 2 & 0 \\
IF & 20 & 5 & 0 \\
\hline
\end{tabular}

the evaluation, the tests were grouped according to their stated levels of sensitivity. In this way 20 borderline samples resulted, combining HA and IF tests. This population gave mean and SD ELISA values of $299 \pm 49 \mathrm{mU}$, corresponding to a distribution ranging, at a $95 \%$ probability, from 201 to $397 \mathrm{mU}$. It is apparent that this population is completely discriminated from the negative one, while some overlap exists with the positive class. A range for the borderline samples could be tentatively assumed to be $200-350 \mathrm{mU}$ (ie, from the negativeborderline cut-off to $\bar{x}+$ SD where $\bar{x}$ refers to the mean ELISA response of HA-IF borderlines).

By applying the same analysis to the 16 samples classified as borderline by DT and cLIA, a mean value of $374 \pm 77 \mathrm{mU}$ and a $95 \%$ probability range 220-528 were obtained. Also in this case no overlap with the negative population can be observed, but borderlines and positives largely superimpose.

The classification of the 86 samples according to the ELISA response is shown in Table 4 as resulting from the above data, while the Figure illustrates the correlations obtained.

Some conclusive considerations can be attempted. In terms of specificity, ELISA looks comparable with the other tests (if not superior) as no sample classified

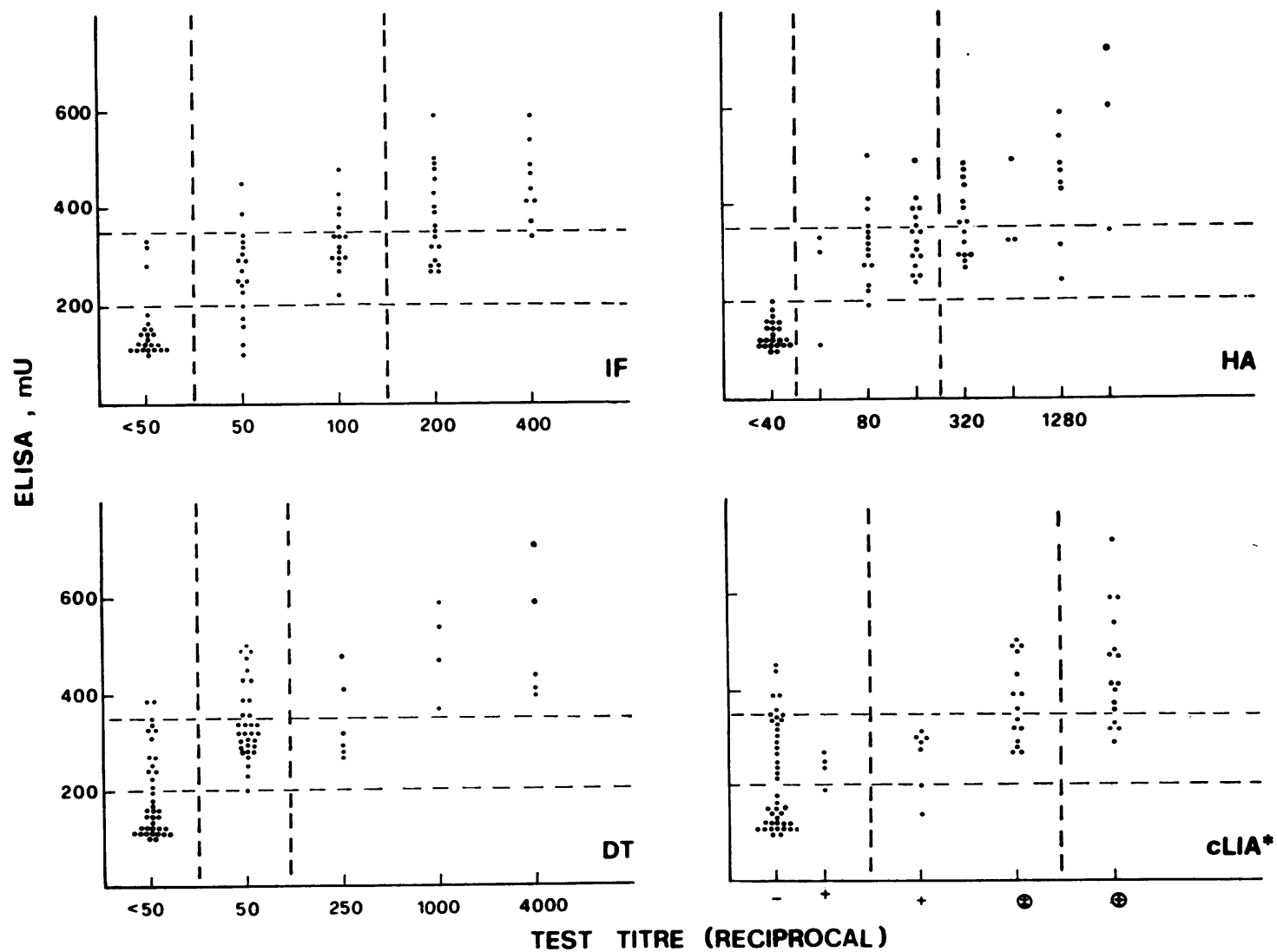

Correlation of the ELISA responses with the results of the reference tests. The class subdivision on each scale (negative, borderline, positive) is indicated by dotted lines.

* The doubtful precipitation arcs, which are indicated as \pm for undiluted and $\bigoplus$ for $1 / 4$ diluted serum, were ascribed to negative and borderline classes, respectively. $\oplus$ represents a positivity with the diluted serum. 
Table 4 Classification of the samples $(n=86)$ according to the ELISA response $(\mathrm{mU})$

\begin{tabular}{llll}
\hline $\begin{array}{l}\text { Sample } \\
\text { classification }\end{array}$ & Frequency & Mean $\pm S D$ & Range \\
\hline Negative & 25 & $126 \pm 18$ & $<200$ \\
Borderline & 34 & $307 \pm 24$ & $200-350$ \\
Positive & 27 & $427 \pm 45$ & $>350$ \\
\hline
\end{tabular}

as negative by all these tests was ELISA-positive, and vice versa; ELISA appears to correlate better with HA and IF tests than with DT and CLIA, as indicated by the frequency in the respective group classifications; in particular, the number of negatives, much lower for ELISA, IF, and HA than for DT and cLIA, suggests that higher sensitivity levels are obtained in the former cases.

\section{Conclusions}

The results of the present study indicate a substantial agreement in group classification of samples between the ELISA developed in our laboratories and conventional serological tests for toxoplasmosis. This holds particularly in respect of indirect immunofluorescence and passive haemagglutination, which are to be regarded as suitable tests. ${ }^{910}$

The ELISA conditions adopted were directed to obtain a high practicability level rather than to exploit the full potential of the method. Work is presently in progress to optimise further the ELISA performances as for sensitivity (incubation time, sample dilution), response precision, and consistency (reagent stability, way of expressing data), and, especially, possibility of discriminating between IgG and IgM antibody classes.

This work was supported in part by the Special
Project on Biomedical Engineering, National Research Council-CNR, sub-project Chim. 2.

\section{References}

${ }^{1}$ Saunders GC, Clinard EH, Bartlett ML, Sanders WM. Application of the indirect enzyme-labelled antibody microtest to the detection and surveillance of animal diseases. J Infect Dis 1977;136:S528-66.

${ }^{2}$ Sever JL, Madden DL. Enzyme linked immunosorbent assay (ELISA) for infectious agents. Meeting held at the National Institutes of Health (Bethesda, Sept 1976). J Infect Dis 1977;136:S258-340.

${ }^{3}$ Voller A, Bartlett A, Bidwell DE. Enzyme immunoassay for parasitic diseases. Trans R Soc Trop Med Hyg 1976; 70:98-106.

4 Voller A, Bidwell DE, Bartlett A, Fleck DG, Perkins M, Oladehin B. A microplate enzyme-immunoassay for toxoplasma antibody. J Clin Pathol 1976;29:150-3.

${ }^{5}$ Walls KW, Bullock SL, English DK. Use of the enzymelinked immunosorbent assay (ELISA) and its microadaptation for the serodiagnosis of toxoplasmosis. J Clin Microbiol 1977;5:273-7.

${ }^{6}$ Nakane PK, Kawaoi A. Peroxidase-labelled antibody: a new method of conjugation. $J$ Histochem Cytochem 1974;22:1084-91.

? Sabin AB, Feldman HA. Dyes as microchemical indicators of a new immune phenomenon affecting a protozoan parasite (Toxoplasma). Science 1948;108:660-3.

${ }^{8}$ Zotti C, Moiraghi Ruggenini A, Massaro L. La cross-over linked immunoassay (CROSS-LIA): nuovo metodo per l'accertamento diagnostico dell'infezione toxoplasmica. Primi risultati e confronto con altre metodologie. XXVIII Congresso Nazionale della Associazione Italiana Patologi Clinici, Sanremo, Italy, 22-25 Giugno.

${ }^{9}$ Goldman M. Staining Toxoplasma gondii with fluoresceinlabelled antibody. 1: the reaction in smears of peritoneal exudate. J Exp Med 1957;105:549-56.

10 Jacobs L, Lunde MN. A hemagglutination test for toxoplasmosis. J Parasitol 1957;43:308.

Requests for reprints to: Professor G Poli, Università di Milano, Facoltà di Medicina Veterinaria, Istituto di Microbiologia e Immunologia, Via Celoria 10, Milan, Italy. 